\title{
Microtubule transport, concentration and alignment in enclosed microfluidic channels
}

\author{
Ying-Ming Huang • Maruti Uppalapati • \\ William O. Hancock • Thomas N. Jackson
}

Published online: 29 December 2006

(C) Springer Science + Business Media, LLC 2007

\begin{abstract}
The kinesin-microtubule system has emerged as a versatile model system for biologically-derived microscale transport. While kinesin motors in cells transport cargo along static microtubule tracks, for in vitro transport applications it is preferable to invert the system and transport cargofunctionalized microtubules along immobilized kinesin motors. However, for efficient cargo transport and to enable this novel transport system to be interfaced with traditional microfluidics, it is important to fabricate enclosed microchannels that are compatible with kinesin motors and microtubules, that enable fluorescence imaging of microtubule movement, and that provide fluidic connections for sample introduction. Here we construct a three-tier hierarchical system of microfluidic channels that links microscale transport channels to macroscopic fluid connections. Shallow microchannels ( $5 \mu \mathrm{m}$ wide and $1 \mu \mathrm{m}$ deep) are etched in a glass substrate and bonded to a cover glass using PMMA as an adhesive, while intermediate channels ( $\sim 100 \mu \mathrm{m}$ wide) serve as reservoirs and connect to $250 \mu \mathrm{m}$ deep microchannels that hold fine gauge tubing for fluid injection. To demonstrate
\end{abstract}

Ying-Ming Huang and Maruti Uppalapati contributed equally to this work.

Electronic Supplementary Material Supplementary material is available in the online version of this article at http://dx.doi.org/10.1007/s10544-006-9019-1

Y.-M. Huang · T. N. Jackson $(\bowtie)$

Center for Thin Film Devices and Department of Electrical

Engineering, Materials Research Institute, Penn State University,

University Park, PA 16802, USA

e-mail:tnj1@psu.edu

M. Uppalapati · W. O. Hancock ( $\square)$

Department of Bioengineering, Penn State University,

University Park, PA 16802, USA

e-mail: wohbio@engr.psu.edu the utility of this device, we first show the performance of a directional rectifier that redirects $96 \%$ of moving microtubules and, because any microtubules that detach rapidly rebind to the motor-coated surface, suffers no microtubule loss over time. Second, we develop an approach, using a headless kinesin construct, to eliminate gradients in motor adsorption and microtubule binding in the enclosed channels, which enables precise control of kinesin density in the microchannels. Finally, we show that a $60 \mu \mathrm{m}$ diameter circular ring functionalized with motors concentrates and aligns bundles of $\sim 3000$ uniformly oriented microtubules, while suffering negligible ATP depletion. These aligned isopolar microtubules are an important tool for microscale transport applications and can be employed as a model in vitro system for studying kinesin-driven microtubule organization in cells.

Keywords Kinesin - Microtubule $\cdot$ Motor proteins · Molecular motors · Microfabrication · Microfluidics · Active transport

\section{Introduction}

In eukaryotic cells, kinesin motor proteins transport intracellular cargo and provide the mechanical forces underlying mitotic spindle morphogenesis and chromosome separation. (Hirokawa et al., 1998; Goldstein and Philp, 1999; Sharp et al., 2000) A continuing challenge in nanoscience is controlling the manipulation and assembly of materials at the nano-scale and the kinesin-microtubule system provides an excellent model system for investigating and exploiting biologically derived nano-scale motion. Conventional kinesin motors move at roughly $1 \mu \mathrm{m} / \mathrm{s}$ and can generate single motor forces in the range of 6-8 $\mathrm{pN}$ in vitro (Svoboda et al., 1993; Meyhöfer and Howard, 1995). These motors are also 
optimal for in vitro investigations because they move long distances $(\sim 1 \mu \mathrm{m})$ along microtubules before detaching, they are reasonably robust proteins, and sufficient amounts of recombinant motors can be bacterially expressed and purified using conventional techniques (Howard et al., 1989; Stock and Hackney, 2001; Brunner et al., 2004).

Although in cells microtubules are generally fixed and motors move along them, for engineered microsystems it is convenient to invert this geometry. In the filament gliding assay, motors are adsorbed to surfaces through their tail domain and microtubules, which can be functionalized with cargo such as biomolecules or nanoparticles, are propelled along the surface by the functional motor heads (Howard et al., 1989; Jia et al., 2004). At present there is an ongoing effort to combine this biological transport system with microfabricated channels to create a novel approach to microscale transport that can supplement existing methods such as pressure driven flow or electrokinetic pumping (Hess et al., 2004; Hancock, 2006). Compared to existing microfluidic transport approaches, the kinesin-microtubule system offers several potential advantages. First, because analytes are bound to microtubules that move along the surface, the transport is independent of bulk flow and can even move against bulk flow and up concentration gradients. Second, this biological transport is fueled by ATP present directly at the site of action, and does not require external power or bulky pumps. However, to harness this biological transport system for microscale devices, such as lab-on-a chip systems, the motors and microtubules must be integrated into microfabricated systems, and the direction of transport must be controlled.

A number of groups have shown that microfabricated surface features such as walls and channels can be used to redirect and control the trajectory of kinesin-driven microtubules (Hiratsuka et al., 2001; Hess et al., 2002; Clemmens et al., 2003; Moorjani et al., 2003). However, in most of these demonstrations the top of the channels are open to the bulk solution and because filaments can detach from the kinesin coated surface and diffuse away, microtubules are lost from the surface over time (half of the observed population in some designs (Clemmens et al., 2004; van den Heuvel et al., 2005)). This makes it difficult to obtain controlled motion over long distances or generate high densities of oriented microtubules, which are requirements for transporting significant amounts of cargo over distances relevant for device applications. Hence, it is important to develop methods for fabricating enclosed microfluidic channels that can be functionalized with motors and which trap the moving microtubules in the channels to prevent loss.

Here we report the fabrication of a microfluidic system composed of enclosed glass microchannels that are designed for observing and controlling kinesin-driven microtubule transport. This confinement geometry (channel cross-section $\sim 5 \mu \mathrm{m} \times \sim 1 \mu \mathrm{m}$ ) enables long-distance movement ( $\sim 5 \mathrm{~mm}$ ) and directional control, prerequisites for developing biomotor-driven transport for hybrid lab-ona-chip devices. We demonstrate the utility of this system by constructing a closed circular ring with inlet and outlet ports and showing that over time it sorts and concentrates many hundreds of uniformly aligned and bundled microtubules. This ability to collect a dense and uniformly aligned bundle of microtubules is not possible with existing techniques, and this system is an important step for microscale transport applications and as a tool for investigating kinesin-microtubule interactions that underlie cell structure and function.

\section{Materials and methods}

\subsection{Enclosed microchannel fabrication}

In order to confine microtubules, we developed an approach for fabricating enclosed microchannels that are compatible with kinesin motors and microtubules, enable fluorescence imaging of microtubule movement, and provide simple fluidic connections for sample introduction. The design consists of a three tier hierarchical structure (Fig. 1) that links microscale transport channels to macroscopic fluid connections. Shallow microchannels $(\sim 5 \mu \mathrm{m}$ wide and $\sim 1 \mu \mathrm{m}$ deep) and related structures for microtubule motility experiments connect to intermediate channels $(\sim 100 \mu \mathrm{m}$ wide) that serve as reservoirs and also connect to $250 \mu \mathrm{m}$ deep macrochannels that hold fine gauge tubing for simple external fluid connections. The micro, intermediate, and macro scale channels are etched in a $1 \mathrm{~mm}$ thick borosilicate glass substrate and bonded to a cover glass using polymethyl methacrylate (PMMA) as an adhesive. Figure 2 illustrates the process flow for fabricating the three tier hierarchical channels.

Before channel fabrication, glass substrates were cleaned by immersion in acetone and then isopropyl alcohol in an ultrasonic bath for $10 \mathrm{~min}$ each, followed by immersion in piranha solution $\left(\mathrm{H}_{2} \mathrm{SO}_{4}: \mathrm{H}_{2} \mathrm{O}_{2}=4: 1\right)$ for $20 \mathrm{~min}$. The piranha treatment both cleans the glass and results in a high-surfaceenergy $\mathrm{OH}$-saturated surface that improves the adhesion of deposited layers. To etch the microscale and intermediate channels, a $10 \mathrm{~nm} \mathrm{Cr}$ layer and a $100 \mathrm{~nm}$ Au layer were deposited on the cleaned glass substrate using a resistive filament thermal evaporator. Channel designs were then patterned in the metal layers by photolithography using a Karl Suss MA-55 aligner with Shipley 1811 photoresist and wet etching. The $\mathrm{Cr} / \mathrm{Au}$ layer eliminates photoresist lifting that can be a problem for direct etching of glass with only a photoresist mask. Using the metal layers as a mask along with the photoresist, the shallow micro and intermediate channels were then etched using a buffered oxide etch solution (BOE, $\mathrm{NH}_{4} \mathrm{~F} / \mathrm{HF} 10: 1$ ) at room temperature with moderate 

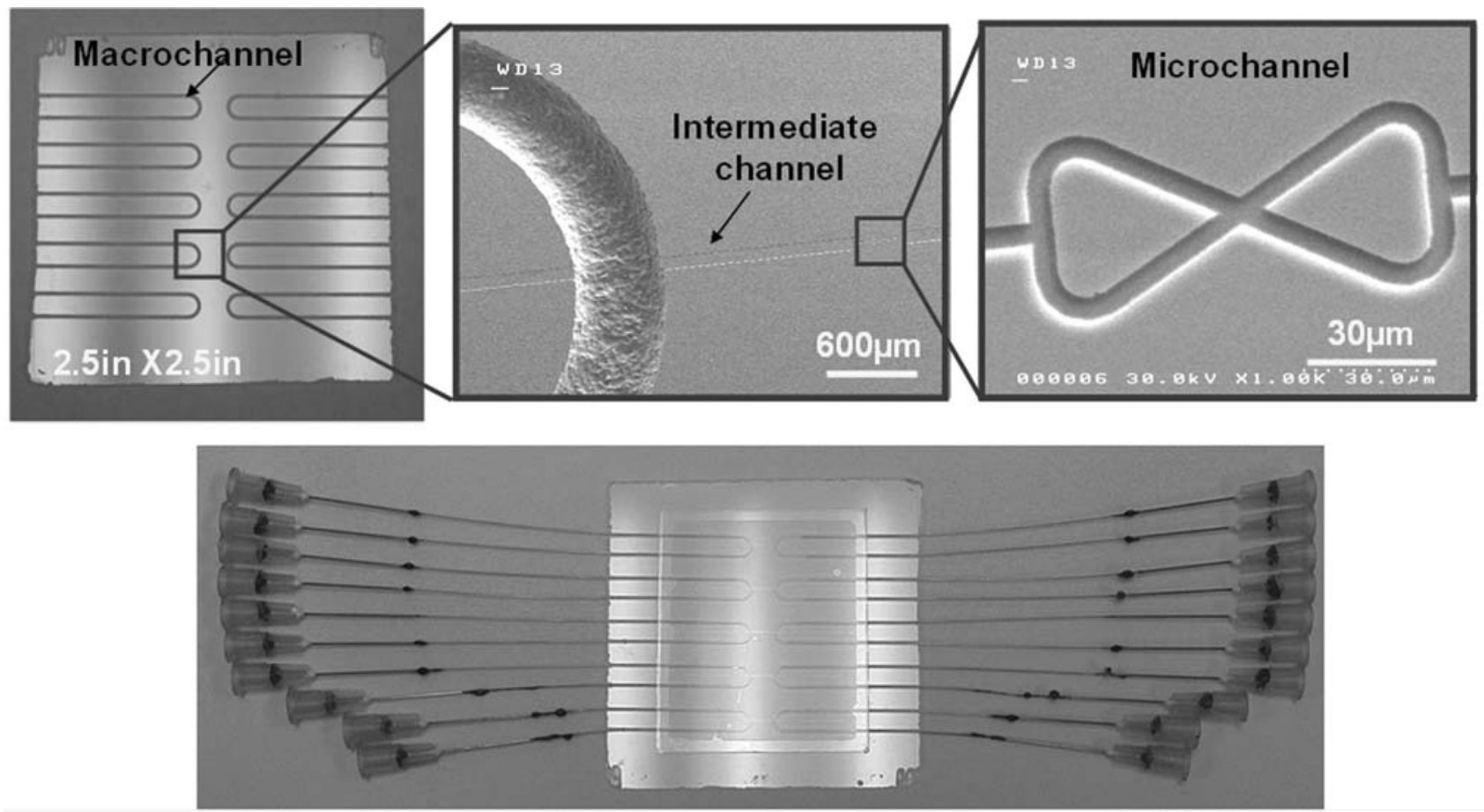

Fig. 1 Hierarchical microchannel design. Macrochannels $(250 \mu \mathrm{m}$ deep) enable sample introduction. Intermediate channels $(100 \mu \mathrm{m}$ wide and $1 \mu \mathrm{m}$ deep) connect to microchannels ( $5 \mu \mathrm{m}$ wide and $1 \mu \mathrm{m}$ deep) where microtubule motility is observed. Bottom panel shows a completed sample including the coverglass bonded using PMMA adhesive, and tubing for sample injection

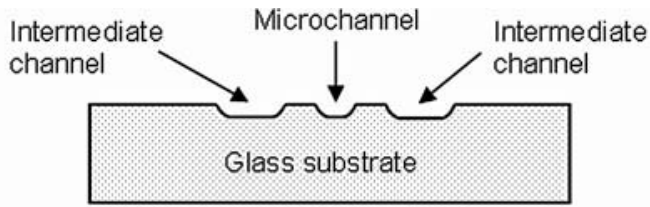

(A)

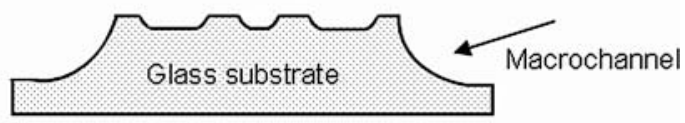

(B)

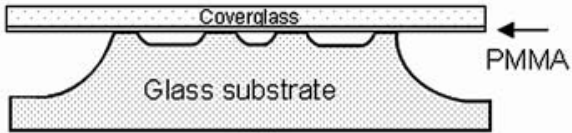

(C)

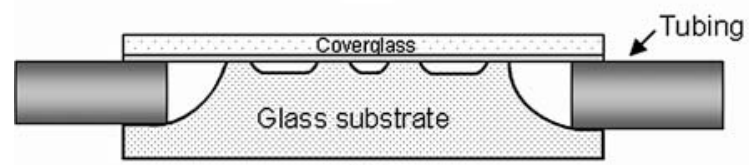

(D)

Fig. 2 Procedure for fabricating three tier hierarchical microchannels. (A) Microchannels and intermediate channels $1 \mu \mathrm{m}$ deep are patterned by photolithography and etched in the glass substrate. (B) Macrochannels $250 \mu \mathrm{m}$ deep are then etched. (C) A layer of PMMA is deposited on the coverglass and the coverglass is bonded to the etched glass substrate to enclose the channels. (D) Stainless steel tubing is inserted into the macro channels and bonded using epoxy, and this tubing is connected to syringe needles for sample introduction agitation. A 20 min etch resulted in $1 \mu \mathrm{m}$ deep channels. Following channel etching, the photoresist, $\mathrm{Au}$, and $\mathrm{Cr}$ layers were stripped by immersing in acetone, Au etchant, and Cr etchant, respectively.

The macroscale channels were also fabricated using a combination photoresist/metal etch mask and were etched into the glass using concentrated (49\%) hydrofluoric acid (HF). Deep glass etching is significantly more challenging than shallow channel etching and defect related pinholes, etch mask lifting, and other problems can be significant (Bien et al., 2003; Bu et al., 2004). To allow defect-free macroscale channel etching, we used a combination of $40 \mu \mathrm{m} \mathrm{SU}-8$ photoresist, $1.5 \mu \mathrm{m}$ electroplated gold (Techni gold 25ES, Technic Inc.) and thermally evaporated layers of $\mathrm{Cr}(10 \mathrm{~nm})$ and $\mathrm{Au}(100 \mathrm{~nm})$. The evaporated $\mathrm{Cr} / \mathrm{Au}$ layer provides good adhesion to the glass substrate and provides a seed layer for gold electroplating. The thick electroplated Au provides good protection against concentrated HF with reduced defect density and cost compared to evaporation or sputtering. The SU-8 photoresist on top of the electroplated Au provides additional protection against pinholes. Etching for $45 \mathrm{~min}$ in $49 \% \mathrm{HF}$ at room temperature results in $\sim 300 \mu \mathrm{m}$ deep macrochannels. After etching, the SU-8 layer was removed by piranha cleaning and the electroplated $\mathrm{Au}$ and evaporated $\mathrm{Cr} / \mathrm{Au}$ layers removed by wet etching. Alternatively, the sample can be simply soaked in Cr etchant to lift off the whole $\mathrm{Cr} / \mathrm{Au} / \mathrm{Au} / \mathrm{SU}-8$ layer. 


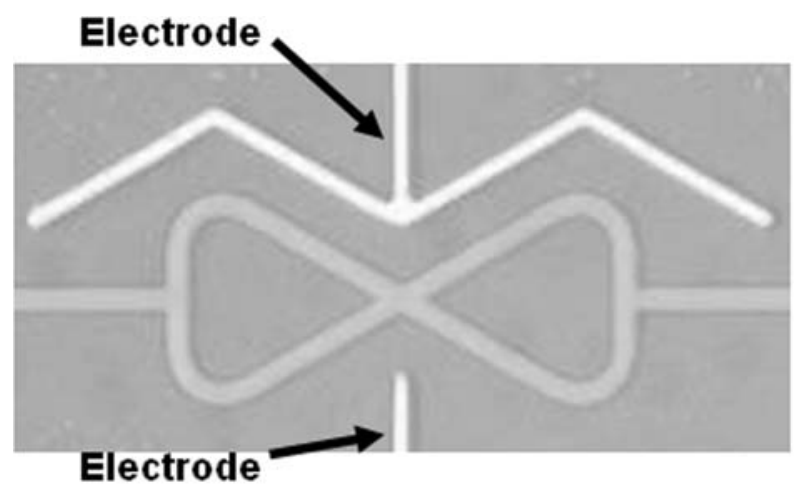

(A)

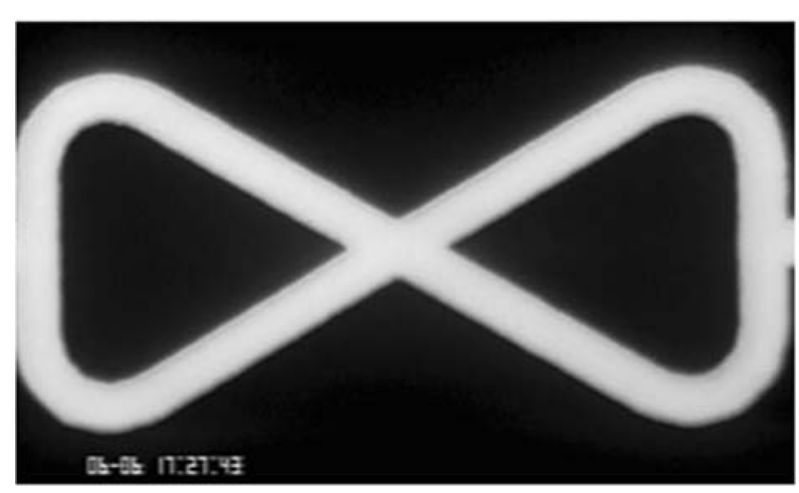

(B)

Fig. 3 Microchannel design and performance. (A) Optical micrograph of enclosed microchannels with embedded electrodes. (B) Fluorescent micrograph of enclosed channels filled with a solution of rhodaminelabeled casein to demonstrate the quality of the bonding between the etched substrate and the coverglass

One advantage of this fabrication method is that electrodes can be integrated into the design along with the micro, intermediate and macro channels. Integrating electrodes opens the possibility of using electric fields to guide microtubules along different paths in channels (Jia et al., 2004; van den Heuvel et al., 2006). To demonstrate the incorporation of electrodes into the design, a $100 \mathrm{~nm}$ Cr layer was deposited onto the engineered glass surface using an Edward E306A sputtering system. Sputtering was used for this step because it provides significantly better coverage over the etched surfaces than thermal evaporation. The Cr layer was then patterned by photolithography using Shipley 1811 photoresist and wet etching. After etching, the photoresist was removed by acetone. Figure 3 shows an example of a microchannel with integrated electrodes.

To cap and enclose the channels, a thin layer of PMMA was used as an adhesive to bond a coverslide to the etched glass substrate. This PMMA bonding approach enables the joining of materials that have different chemical compositions and expansion coefficients, is more tolerant of partic- ulates than anodic bonding or fusion bonding, and can be carried out at lower temperatures (Bilenberg et al., 2004). In control experiments we found that PMMA is compatible with kinesin motors (which functionally adsorb to it) and microtubules (which do not nonspecifically adsorb in the presence of casein). PMMA also has very low autofluorescence at wavelengths of interest for fluorescent markers. $996 \mathrm{kDa}$ PMMA was chosen because it is sufficiently adhesive at convenient bonding temperatures to bond the two layers and sufficiently viscous to prevent filling of the $1.5 \mu \mathrm{m}$ deep channels. A $500 \mathrm{~nm}$ thick PMMA layer was spin-coated from chlorobenzene on $48 \mathrm{~mm} \times 50 \mathrm{~mm}$ coverglass (Gold seal $\mathbb{R}$ ) \#1, thickness: $0.13-0.17 \mathrm{~mm}$ ) and baked on a $180^{\circ} \mathrm{C}$ hot plate for 20 min to remove the solvent and flow the PMMA layer. Both the PMMA and glass micro-channel surfaces were then exposed to oxygen plasma for $1 \mathrm{~min}$ to increase the adhesion (Farrens et al., 2005). Finally the PMMA coated coverslip and glass substrates were laminated using a Warner 100 hydraulic press laminator at $50 \mathrm{psi}$ and $120^{\circ} \mathrm{C}$ for $10 \mathrm{~min}$, and cooled to room temperature under pressure.

To enable sample introduction, fine gauge stainless steel tubing (O.D. $230 \mu \mathrm{m}$ ) is inserted into the macrochannels and sealed with epoxy. Hypodermic needles are bonded to the fine tubing with epoxy, and connected by luer fittings to syringes containing motor and microtubule solutions. Figure 1 shows electron micrographs of the channels and a photo of the finished devices containing input tubing. In Fig. 3(A), a microchannel with incorporated electrodes suitable for electrical stimulation of the sample is shown, and in Fig. 3(B), a solution of rhodamine-labeled casein is introduced into the channel and fluorescently imaged to demonstrate that the coverglass bonds well to the patterned substrate with no leakage.

\subsection{Kinesin and microtubules}

Full-length hexaHis-tagged Drosophila conventional kinesin was used for all motility experiments (Yang et al., 1989). In some experiments, headless kinesin was used, which is a modified kinesin construct that contains the rod and tail domains and a hexaHis tag, but lacks its motor domain (Hancock and Howard, 1998). All motors were expressed in bacteria and purified by $\mathrm{Ni}$ column chromatography as previously described (Hancock and Howard, 1998; Coy et al., 1999). Tubulin was purified from bovine brains and labeled with rhodamine as previously described (Williams and Lee, 1982; Hyman et al., 1991). Microtubules were polymerized by mixing $32 \mu \mathrm{M}$ rhodamine-labeled tubulin, $4 \mathrm{mM} \mathrm{MgCl}{ }_{2}$, $1 \mathrm{mM} \mathrm{GTP}$ and 5\% DMSO in BRB80 buffer ( $80 \mathrm{mM}$ PIPES, 1 mMEGTA, $1 \mathrm{mM} \mathrm{MgCl}_{2}$, pH 6.9 with $\mathrm{KOH}$ ), incubating at $37^{\circ} \mathrm{C}$ for $20 \mathrm{~min}$, and then diluting into a solution containing $10 \mu \mathrm{M}$ paclitaxel. 
To immobilize motors inside the microchannels, a solution containing $4 \mathrm{mg} / \mathrm{ml}$ casein, $7.5 \mu \mathrm{g} / \mathrm{ml}$ conventional kinesin and (in some cases) $32 \mu \mathrm{g} / \mathrm{ml}$ headless kinesin in BRB80 buffer was introduced. This solution was flushed with a second solution containing $0.64 \mu \mathrm{M}$ rhodaminelabeled microtubules, $10 \mathrm{mM}$ ATP, $50 \mu \mathrm{M}$ paclitaxel and antifade reagents $(0.1 \mathrm{M}$ D-glucose, $0.1 \mathrm{mg} / \mathrm{ml}$ glucose oxidase, $0.04 \mathrm{mg} / \mathrm{ml}$ catalase and $0.35 \mathrm{M} \beta$-mercaptoethanol) in BRB80 buffer. These concentrations are five to twenty times higher than our standard levels (Moorjani et al., 2003), to compensate for the low volume to surface area of the microchannels.

\subsection{Microscopy and image analysis}

Movement of the confined microtubules was observed by epifluorescence microscopy (Nikon E600, $100 \times 1.3$ N.A. objective). Images recorded on videotape were digitized using Scion Image (Scion Corporation). The intensity values were then corrected for the gamma function of the camera (Genwac GW-902H) and the background noise was subtracted using Image J. To estimate the microtubule density in the microchannels, the integrated fluorescence intensity across the channel was measured and compared to the intensity of single isolated microtubules.

\section{Results and discussion}

While there has been extensive work using patterned surfaces to redirect kinesin-driven microtubule transport (Hiratsuka et al., 2001; Hess et al., 2002; Moorjani et al., 2003), three dimensional confinement is a prerequisite for harnessing this biological system for microscale transport applications and for combining motor driven transport with pressuredriven microfluidics. Although kinesins immobilized on engineered surfaces can faithfully direct microtubules and their associated cargo, diffusion and convection in the bulk solution above the surface can easily cancel out any motordriven transport. Hence, to achieve kinesin-driven microtubule transport in enclosed channels, the channels must be fabricated in a transparent and motor compatible material, the architecture must enable sample introduction and visualization, and the channels must redirect microtubules without clogging or running out of fuel. Below we describe a simple redirection pattern that demonstrates the utility of these channels, and we then describe a novel method to optimize the uniformity of motor adsorption in the channels. Finally, we present a circular ring structure that enables long term visualization of microtubule motility and acts to concentrate a large population of uniformly oriented and aligned microtubules. This level of control is an important step towards utilizing the kinesin-microtubule system for microscale transport, and it is a significant advance beyond previous approaches (Huang et al., 2005). Furthermore, obtaining this level of control over microtubule transport and assembly is an important enabling tool for using refined systems to study the role of motors and microtubules in complex cellular processes such as axonal transport and mitosis.

\subsection{Enclosed microchannels redirect moving microtubules}

The first design we analyzed is a directional rectifier that passes microtubules traveling in one direction and reverses microtubules moving in the opposite direction. In these gliding assays, kinesins are immobilized in all orientations, but because they contain a flexible domain between their tail and their heads (Hunt and Howard, 1993), they are able to swivel and interact with microtubules only in their preferred stereospecific orientation. Hence, the initial direction that microtubules move depends solely on the orientation in which they land on the surface. Because the persistence length of microtubule (5.2 mm (Gittes et al., 1993)) is very long compared to the mean distance between motors adsorbed on surface $(<100 \mathrm{~nm})$, the microtubule are expected (Gibbons et al., 2001) and found to travel in a straight line in the absence physical barriers. However, when microtubules encounter physical barriers such as channel walls, the motor forces cause the microtubule to buckle and be redirected.

A number of groups have shown that three dimensional patterns on motor-coated surfaces will bend or buckle microtubules and reorient their direction (Hiratsuka et al., 2001; Hess et al., 2002; Clemmens et al., 2003; Moorjani et al., 2003; van den Heuvel et al., 2005). However, because the channels are open on their top, detachment from the surface prevents complete redirection and requires an influx of new microtubules to compensate for microtubules lost from the surface. To transform bidirectional motion into unidirectional motion, we designed a rectification pattern (Fig. 4) that permits microtubules entering from one end to pass through, while redirecting microtubules moving from opposite end. Figure 4(A) shows representative paths of two microtubules in the rectifier pattern. Filaments entering from the right (grey traces) buckle at the base of the rectifier, travel leftward in one or the other arms and exit from the left, while microtubules entering from the left (black traces) continue through the entire circuit and exit from the left. To show the rectification ability of the structure, microtubules were introduced from left entrance and observed traveling through the pattern; $96 \%(N=50)$ of microtubules entering from the left were successfully redirected and exited to the left. Importantly, even though microtubules occasionally detach from the motor-coated surface, they remain in the channel and reattach within a few seconds. Also the small confinement geometry $(5 \mu \mathrm{m} \times 1 \mu \mathrm{m})$ prevents the dissociated microtubules from reorienting in the opposite direction. Movement is observed 


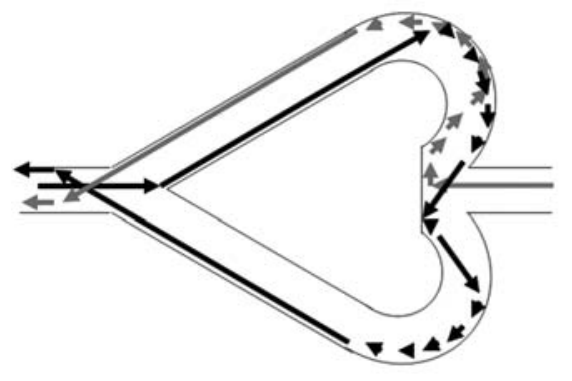

(A)

Fig. 4 Directional rectifier constructed using enclosed microfabricated channels. (A) Expected paths of microtubules in the rectifier pattern (grey traces follow microtubules moving in desired direction, black traces follow microtubules moving in opposite direction).

on not only the bottom and sidewalls of the channel, which are glass, but also the top, which is coated with a layer of PMMA.

Van den Heuvel et al. recently reported a method for measuring electrically driven and motor driven movement of microtubules in enclosed glass microchannels (van den Heuvel et al., 2006). Their fabrication approach consisted of etched glass channels bonded to a top coverglass using a spin-on sodium silicate layer as an adhesive, and they describe a different design for a redirection pattern. Although the specific performance of their rectifier was not described, there is clearly a large design space for creating microchannel patterns that redirect microtubules.

\subsection{Controlling motor density in enclosed channels}

In the microtubule gliding assay the characteristics of microtubule transport are influenced by the density of kinesin motors immobilized on the surface. While the transport speed is virtually unchanged over three orders of magnitude of motor density, the rate that microtubules land on the motorfunctionalized surface and the distance they move before detaching and diffusing away both vary strongly with the motor density (Howard et al., 1989; Hancock and Howard, 1998). Furthermore, there is an optimal motor surface density for redirecting kinesin-driven microtubules using microfabricated walls. At low motor densities collisions with walls tend to cause microtubules to detach from the surface and diffuse away, while at high motor densities microtubules stall when encountering sharp features and vertical walls that, which are needed for proper redirection (Moorjani et al., 2003). Compared to open top channels, enclosed microchannels reduce the impact of both of these factors. At low motor densities, microtubules that detach can rapidly rebind. Further, because motors are present on all internal surfaces and there are few sharp edges, microtubules rarely stall and are faithfully redirected even at very high motor densities.

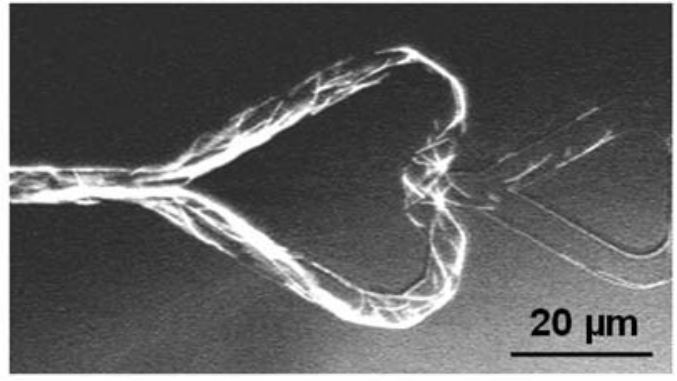

(B)

(B) Microtubules entering from reservoir at left (not shown) travel down either arm and continue through to the other arm, reversing their direction. Microtubules entering from the right collide with the base of the structure and are directed into one of the two arms

However, while motor adsorption is homogeneous with open channels, the high surface to volume ratio of enclosed channels results in motors preferentially adsorbing to the upstream end, creating a gradient in motor adsorption along the channels. High uniform motor densities can be achieved throughout the device by flowing large volumes of motor solution through the channels. However, high motor densities in the intermediate channels result in rapid attachment of microtubules at the junction between the macro channel and the intermediate channel. The junction thus acts as a filter, preventing microtubules from reaching the downstream end of the intermediate channel and the microchannel. Figure 5(A) shows the steep gradient of microtubule binding in the intermediate channel adjacent to the macro channel junction. Because this filtering effect is more severe for long microtubules, only short microtubules are seen in the intermediate channels (Fig. 5(A)). Over time, the long microtubules form a dense network that prevents any microtubules from entering the intermediate channel.

We solved this motor gradient problem using a modified kinesin that includes the rod and tail domains that mediate motor interactions with surfaces, but lacks the motor domain and hence does not bind microtubules (Hancock and Howard, 1998). When this headless kinesin protein is introduced into the enclosed channels along with the full-length functional motors, it competes for surface binding with the functional motors, and hence limits the number of functional motors that can adsorb onto the surface. When $32 \mu \mathrm{g} / \mathrm{ml}$ headless motors were combined with $7.5 \mu \mathrm{g} / \mathrm{ml}$ functional kinesin motors and flushed into the channels, the functional motor density was optimal for long distance transport and no motor gradient was apparent (Fig. 5(B)). Hence, despite the high surface area inherent in these small enclosed microchannels, by varying the ratio of headless motors to functional motors, the functional motor density can be specified over a wide range of densities and optimal motility can be achieved throughout all regions of the device. 


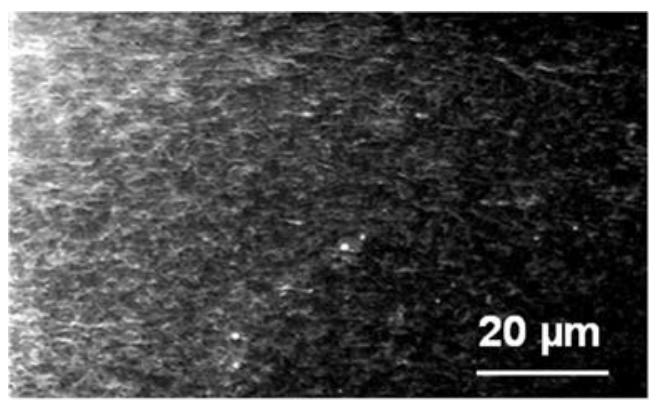

(A)

Fig. 5 Eliminating kinesin motor gradients using a headless kinesin construct. (A) Image of an intermediate channel when only full-length motors are used. The microtubules bind avidly to the macro channel (to the left of the intermediate channel) and the proximal portion of the intermediate channel (left side of the image). The number of micro-

\subsection{Long term confinement and concentration of microtubules in a circular ring}

In harnessing microtubule based transport and in developing experimental models of complex cellular processes, one recurring challenge has been concentrating significant numbers of microtubules that are parallel and uniformly oriented. A number of approaches have been investigated including immobilizing short microtubule seeds and growing filaments preferentially off of one end (Brown and Hancock, 2002), binding microtubules to functionalized surfaces through one end and using flow to push them onto the surface (Limberis et al., 2001), and reorienting kinesin-driven microtubules using fluid flow or electric fields (Stracke et al., 2002; Prots et al., 2003; van den Heuvel et al., 2006). Furthermore, microfabricated open top structures have been shown to assemble moderate numbers of parallel and uniformly oriented microtubules (Hess et al., 2002; Clemmens et al., 2004; Lin et al., 2006), and the rectifier described above acts to collect uniformly oriented filaments. However, we sought to create a channel geometry that sorts and concentrates dense bundles of uniformly oriented microtubules as a tool for transport applications and to mimic cellular microtubule architectures.

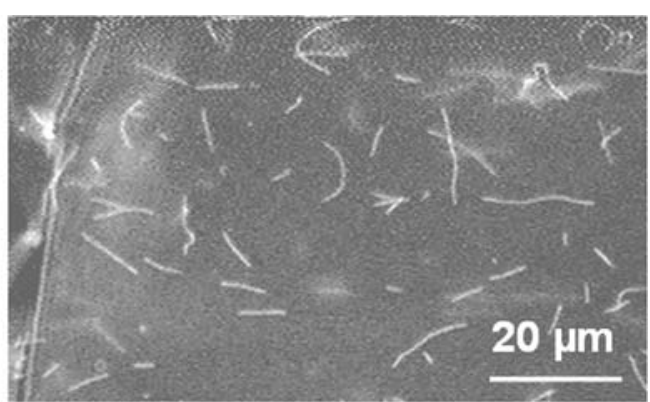

(B)

tubules entering microchannels (located to the right of the intermediate channel) is therefore very small. (B) When full-length kinesin motors are combined with headless kinesin, the motor density is reduced and the gradient in the intermediate channel is eliminated. The edge of the macro channel can be seen at left

We created a microtubule storage ring designed to collect and concentrate uniformly aligned microtubules driven by kinesin motors (Fig. 6). As with the directional rectifier, motors were adsorbed and observed to be functional on all interior surfaces. Blocking motor activity on the side walls, a prerequisite for open channels (Hiratsuka et al., 2001; Moorjani et al., 2003; Cheng et al., 2005), is not required for enclosed channels. The design consists of a circular ring with entry and exit channels that connect to reservoirs (intermediate channels) $100 \mu \mathrm{m}$ wide where microtubules are introduced. Microtubules that are transported into the $60 \mu \mathrm{m}$ diameter circular track from the adjoining reservoir are guided in a counterclockwise direction by the curved walls, while those moving clockwise typically exit the structure within one half revolution (Fig. 7, also see accompanying movie). Microtubules that detach from the surface quickly rebind and continue movement. Rectifiers are included on each end of the ring to return any microtubules that escape the ring (Fig. 6). Because microtubules continuously enter from the reservoirs at either end, the number of oriented microtubules builds over time, resulting in a dense bundle of aligned microtubules after $30 \mathrm{~min}$, much higher density than has been demonstrated for patterns in non-enclosed
Fig. 6 SEM image of the microtubule storage ring. Microtubules originate in the intermediate channels and are transported by immobilized kinesins into the microchannels. The microchannels are designed so that filaments traveling counterclockwise in the ring are retained, while any traveling clockwise exit the ring, are reversed by the rectifiers, and return in a counterclockwise orientation

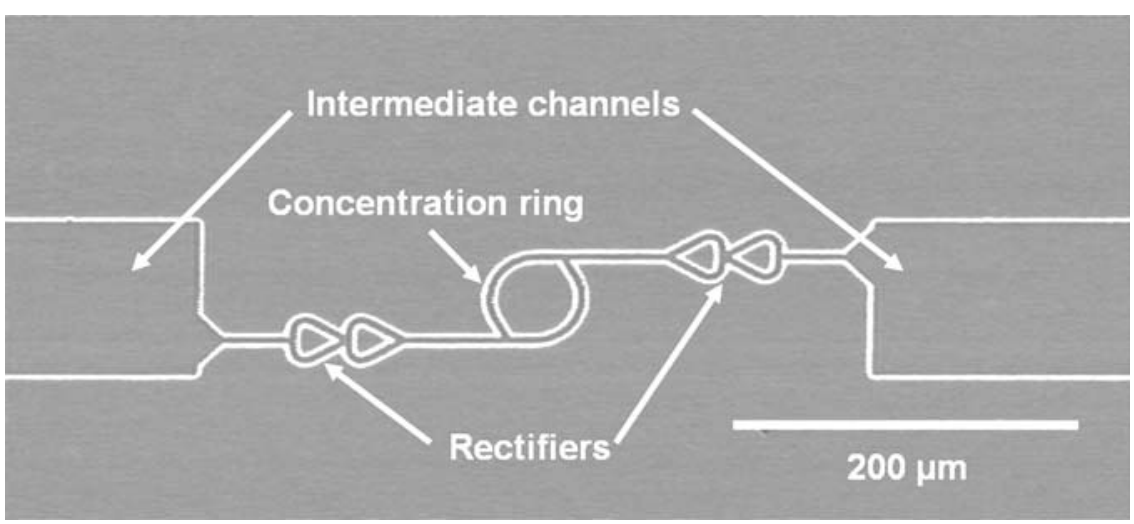




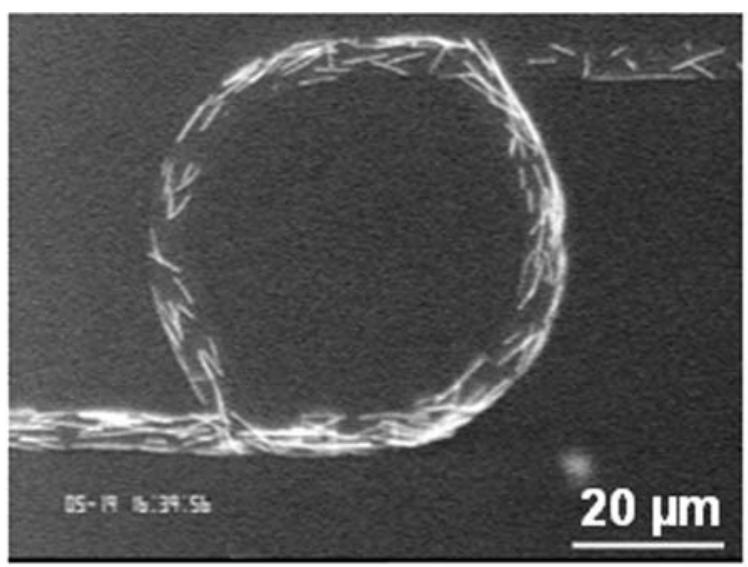

(A)

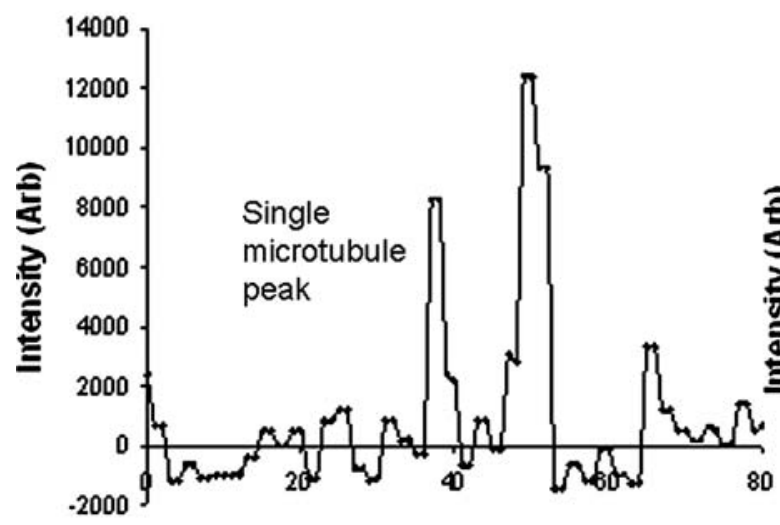

Pixel

(C)

Fig. 7 Microtubule movement and concentration in a microfabricated ring. (A) Microtubule movement shortly after injecting microtubules into the device. Microtubules are moving in both directions in this case. (B) Image of concentration ring after 90 min of accumulation; almost all microtubules are moving counterclockwise in this case. (C) and (D)

undercut channels (Clemmens et al., 2004; Lin et al., 2006). The structure can be continually monitored under the microscope, and we found that microtubules are retained and continue to move for more than $90 \mathrm{~min}$.

Early after introducing microtubules, individual filaments can be observed being guided around the ring, but at later times the microtubules are sufficiently dense to prevent simple counting. To determine the number of microtubules present after $90 \mathrm{~min}$, the fluorescence intensity was integrated across the channel, and the value divided by the integrated intensity of an individual microtubule (see Methods for details). This image analysis indicated that the ring cross section contains $162 \pm 40$ microtubules (mean $\pm \mathrm{SD}, N=6$ cross-sections). Assuming an average microtubule length of $10 \mu \mathrm{m}$, the total number of microtubules in the ring is approximately 3000. Based on their typical speed of $\sim 0.7 \mu \mathrm{m} / \mathrm{s}$, microtubules present in the ring for the entire 90 min move a distance of $\sim 4 \mathrm{~mm}$.

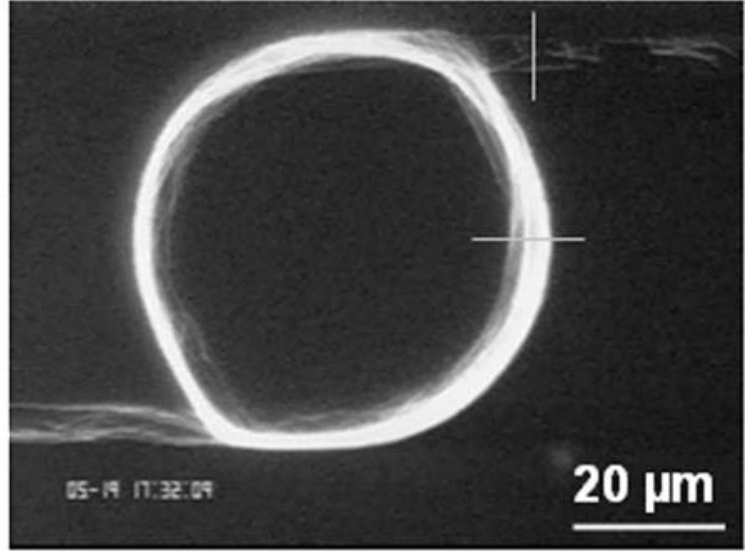

(B)

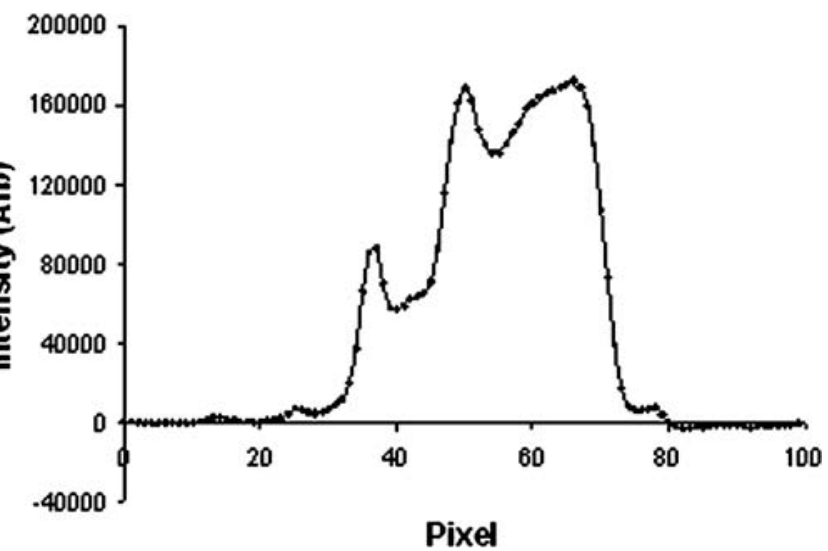

(D)

Fluorescence intensity profiles taken along lines in panel B, for estimating the number of microtubules in the ring. Single microtubule peaks in panel $\mathrm{C}$ were obtained in a sparsely populated area, and together with the integrated fluorescence cross-section in panel D used to calculate the number of microtubules in the ring

A second intriguing observation was that despite the large surface to volume ratio in the channel, the kinesin-driven transport continued for over $90 \mathrm{~min}$, meaning the ATP fuel is not being used up. The microtubule gliding velocity in the ring shown in Fig. 7, was $738 \pm 62 \mathrm{~nm} / \mathrm{s}$ (mean $\pm \mathrm{SD}$, $N=25$ ) shortly after introducing the microtubules and 702 $\pm 58 \mathrm{~nm} / \mathrm{s}$ after $90 \mathrm{~min}$. Data from Schief et al. (2004), who measured the gliding velocity of conventional kinesin at a range of ATP and ADP concentrations, indicate that with an initial concentration of $10 \mathrm{mM}$ ATP, conversion of ATP to ADP will cause a proportional decrease in the gliding velocity. This small reduction after $90 \mathrm{~min}$ of operation implies there is a $\sim 5 \%$ decrease in ATP from $10 \mathrm{mM}$ to $9.5 \mathrm{mM}$.

Because of the small volumes and continuous consumption of ATP by the motors, ATP depletion (and the accompanying ADP buildup) would be expected to be a limiting factor in the lifetime of this device. However, we show here 
that this is not the case because diffusion of ATP from the reservoirs formed by the intermediate channels is sufficient to replenish ATP consumed in the microchannel ring. The first question is: at the 90 min time point when $\sim 3000$ microtubules are in the ring, what is the ATP consumption rate? By comparison to standard microtubule gliding assays in flow cells (Hancock and Howard, 1998), we estimate that microtubules are being propelled by roughly 10 motors each. Biochemical assays have shown that conventional kinesin hydrolyzes approximately 100 ATP per second when walking along microtubules and roughly 0.01 ATP per second when not interacting with microtubules (Hackney, 1994). This low fuel consumption when motors are not engaged with microtubules is one important factor slowing the ATP depletion. The volume of the ring (a $60 \mu \mathrm{m}$ diameter annulus of width $\sim 5 \mu \mathrm{m}$ and depth $\sim 1 \mu \mathrm{m}$ ) is approximately $1 \mathrm{pL}$, and the initial ATP concentration is $10 \mathrm{mM}$, meaning the ring contains $10 \mathrm{fmol}$ or $6 \times 10^{9}$ molecules of ATP. For 3000 microtubules with ten motors moving each microtubule, the motors would be predicted to burn $3 \times 10^{6}$ molecules of ATP per second, which means that without replenishment all of the ATP would be hydrolyzed in roughly $30 \mathrm{~min}$.

In our design, the circular ring is connected to intermediate channel reservoirs (100 $\mu \mathrm{m}$ wide and $2 \mathrm{~mm}$ long) by microchannels that are $5 \mu \mathrm{m}$ wide, $1 \mu \mathrm{m}$ deep and $200 \mu \mathrm{m}$ long. Given the ATP consumption rate calculated above, we can calculate that diffusion of ATP through this $200 \mu \mathrm{m}$ connecting channel is sufficient to maintain high concentrations in the ring. Using a diffusion constant $D=3 \times 10^{-10} \mathrm{~m}^{2} / \mathrm{s}$ for ATP (Rostovtseva and Bezrukov, 1998) and taking a maximal concentration gradient of $10 \mathrm{mM}$ in the intermediate channel and zero in the ring, the maximum flux from the reservoirs into the ring is $9 \times 10^{7} \mathrm{ATP} / \mathrm{s}$, which is 30-times the estimated consumption rate calculated above. Therefore, a concentration gradient of $0.3 \mathrm{mM}$ (leaving a steady-state concentration of $9.7 \mathrm{mM}$ ATP in the ring) should be sufficient to balance the consumption of ATP in the ring with diffusional flux of ATP from the reservoir. Because the reservoir volume is 200 times the ring volume, and the macrochannel volume is 2500 times the reservoir volume, ATP depletion should not be a problem in operation of this device.

In addition to diffusion of ATP, small pressure changes in the tubing and macrochannels can cause flow from the reservoirs into the small bore microchannels, replenishing the ATP supply. Based on the $3 \times 10^{6} \mathrm{ATP} / \mathrm{s}$ consumption rate calculated above, a flow of $10 \mathrm{fL} / \mathrm{s}$, corresponding to a linear velocity of $2 \mu \mathrm{m} / \mathrm{s}$ in the $5 \mu \mathrm{m}^{2}$ microchannels, would be sufficient to maintain a concentration of $9.5 \mathrm{mM}$ ATP in the ring. However, because microtubules that transiently detach from the surface move slower than the $\sim 0.7 \mu \mathrm{m} / \mathrm{s}$ motordriven rate (see accompanying movie), we do not believe that this degree of bulk flow is present during our measurements, although we cannot rule out the possibility that flow plays a role in ATP replenishment. In either case the heirarchical design, consisting of microchannels with a small active volume connected to intermediate and macrochannels, ensures that active motors in the enclosed microchannels are provided with sufficient fuel for long-term operation.

\section{Conclusion}

The kinesin-microtubule system is an intriguing model for biologically driven transport in engineered microenvironments. In cells these motors provide long distance transport of intracellular cargo, and by immobilizing them on surfaces, long distance microtubule transport can be achieved in vitro under a range of experimental conditions. To harness this transport system for lab-on-a-chip analytical devices, it is necessary to encapsulate the motors and microtubules in enclosed microchannels. This work provides a simple and robust strategy for fabricating these microdevices, and demonstrates that kinesin function, microtubule stability, and sufficient ATP levels are maintained in these systems. Furthermore, novel geometries such as concentration rings enable control of microtubule transport that cannot be achieved using the open top channels employed in most previous studies. Finally, by gaining improved control over the movement, orientation, and number of microtubules, these systems provide novel tools that can be used to probe fundamental issues in subcellular organization. For example, an aligned bundle of microtubules mimics the axon of neurons, which are filled with aligned microtubules to enable intracellular transport to and from the synapse. The mitotic spindle is another structure consisting of a pair of opposed bundles of uniformly oriented microtubules. By combining microtubule binding proteins and other molecular motors with these aligned microtubules, it will be possible to build refined analogs of these cellular structures to test minimal models of their assembly and maintenance.

Acknowledgments This project was funded by the Penn State Center for Nanoscale Science (NSF MRSEC DMR0213623) and by an NSF Biophotonics Grant (0323024) to W.O.H. and T.N.J. funded jointly by NSF and NIH/NIBIB.

\section{References}

D.C.S. Bien, P.V. Rainey, S.J.N. Mitchell, and H.S. Gamble, J. Micromech. Microeng. 13, S34 (2003).

B. Bilenberg, T. Nielsen, B. Clausen, and A. Kristensen, J. Micromech. Microeng. 14, 814 (2004)

T.B. Brown and W.O. Hancock, Nano Lett. 2, 1131 (2002).

C. Brunner, K.H. Ernst, H. Hess, and V. Vogel, Nanotechnology 15, S540 (2004).

M.Q. Bu, T. Melvin, G.J. Ensell, J.S. Wilkinson, and A.G.R. Evans, Sensors and Actuators A-Physical 115, 476 (2004). 
L.J. Cheng, M.T. Kao, E. Meyhöfer, and J. Guo, Small 1, 409 (2005).

J. Clemmens, H. Hess, R. Doot, C.M. Matzke, G.D. Bachand, and V. Vogel, Lab. Chip. 4, 83 (2004).

J. Clemmens, H. Hess, R. Lipscomb, Y. Hanein, K. Bohringer, C. Matzke, G. Bachand, B. Bunker, and V. Vogel, Langmuir 19, 10967 (2003).

D.L. Coy, M. Wagenbach, and J. Howard, J. Biol. Chem. 274, 3667 (1999).

S. Farrens, V. Dragoi, R. Pelzer, M. Wimplinger, and P. Lindner, 207th Meeting of the Electrochemical Society (Quebec, Canada, Electrochemical Society Inc., Pennington, NJ, 2005).

F. Gibbons, J.F. Chauwin, M. Desposito, and J.V. Jose, Biophys. J. 80, 2515 (2001).

F. Gittes, B. Mickey, J. Nettleton, and J. Howard, J. Cell Biol. 120, 923 (1993).

L.S. Goldstein and A.V. Philp, Annu. Rev. of Cell Dev. Biol. 15, 141 (1999).

D.D. Hackney, J. Biol. Chem. 269, 16508 (1994).

W.O. Hancock, Protein-based nanotechnology: Kinesin-microtubule driven systems for bioanalytical applications. Nanodevices for Life Sciences. (C. Kumar. Weinheim, Germany, Wiley-VCH, 2006) vol: 4, p. 241.

W.O. Hancock, and J. Howard, J. Cell Biol. 140, 1395 (1998).

H. Hess, G.D. Bachand, and V. Vogel, Chemistry 10, 2110 (2004).

H. Hess, J. Clemmens, C. Matzke, G. Bachand, B. Bunker, and V. Vogel, Appl. Phys. A-Mater. Sci. \& Process. 75, 309 (2002).

Y. Hiratsuka, T. Tada, K. Oiwa, T. Kanayama, and T.Q. Uyeda, Biophys. J. 81, 1555 (2001).

N. Hirokawa, Y. Noda, and Y. Okada, Curr. Opin. Cell Biol. 10, 60 (1998).

J. Howard, A.J. Hudspeth, and R.D. Vale, Nature 342, 154 (1989).
Y.M. Huang, M. Uppalapati, W.O. Hancock, and T.N. Jackson, IEEE Adv. Packaging 28, 564 (2005).

A.J. Hunt and J. Howard, Proc. Nat. Acad. Sci. USA 90, 11653 (1993).

A. Hyman, D. Drechsel, D. Kellogg, S. Salser, K. Sawin, P. Steffen, L. Wordeman, and T. Mitchison, Methods Enzymol. 196, 478 (1991).

L. Jia, S.G. Moorjani, T.N. Jackson, and W.O. Hancock, Biomedical Microdevices 6, 67 (2004).

L. Limberis, J.J. Magda, and R.J. Stewart, Nano Letters 1, 277 (2001).

C.-T. Lin, M.-T. Kao, K. Kurabayashi, and E. Meyhöfer, Small 2, 281 (2006).

E. Meyhöfer, and J. Howard, Proc. Nat. Acad. Sci. USA 92, 574 (1995).

S.G. Moorjani, L. Jia, T.N. Jackson, and W.O. Hancock, Nano Letters 3, 633 (2003).

I. Prots, R. Stracke, E. Unger, and K.J. Bohm, Cell Biol. Int. 27, 251 (2003).

T.K. Rostovtseva and S.M. Bezrukov, Biophys. J. 74, 2365 (1998).

W.R. Schief, R.H. Clark, A.H. Crevenna, and J. Howard, Proc. Nat. Acad. Sci. USA 101, 1183 (2004).

D.J. Sharp, G.C. Rogers, and J.M. Scholey, Biochi. Et. Biophys. Acta. 1496, 128 (2000).

M.F. Stock and D.D. Hackney, Methods Mol. Biol. 164, 43 (2001).

R. Stracke, K.J. Bohm, L. Wollweber, J.A. Tuszynski, and E. Unger, Biochem. Biophys. Res. Commun 293, 602 (2002).

K. Svoboda, C.F. Schmidt, B.J. Schnapp, and S.M. Block, Nature 365, 721 (1993).

M.G. van den Heuvel, C.T. Butcher, R.M. Smeets, S. Diez, and C. Dekker, Nano. Lett. 5, 1117 (2005).

M.G. van den Heuvel, M.P. de Graaff, and C. Dekker, Sci 312, 910 (2006).

R.C. Williams, Jr. and J.C. Lee, Methods Enzymol 85 Pt B, 376 (1982).

J.T. Yang, R.A. Laymon, and L.S. Goldstein, Cell 56, 879 (1989). 